\title{
ARTIFICIAL INTELLIGENCE IN REAL-TIME EVALUATING ELECTRICAL CONDUCTIVITY OF GREENHOUSE SUBSTRATE
}

\author{
Junning Zhang, Minzan Li, Dexiu Kong, Qizhang Zou \\ Key Laboratory of Modern Precision Agriculture System Integration Research, \\ China Agricultural University, Ministry of Education, P. R. China \\ No.17 Qinghua Donglu, Beijing, P. R. China, 100083 \\ limz@cau.edu.cn
}

Abstract: To support decision-making in precision management of greenhouse cultivation, a portable electrical conductivity detector was developed to realtime measure substrate in greenhouse. It mainly included three units, probes, controlling and monitoring unit, and data processing and displaying unit. The probes were designed based on Four-electrode method. The controlling and monitoring unit provided a constant current into substrate and then measured the voltage drop between two points of substrate. Performance experiments under different conditions were conducted. The result revealed that, when water content was lower than $50 \%$, the substrate electrical conductivity would be affected by both salinity and moisture in substrate. Therefore it was necessary to consider the factor of moisture when evaluating substrate property by substrate electrical conductivity. When water content was higher than $50 \%$, substrate electrical conductivity was mainly affected by substrate salinity, and the effect of moisture could be ignored.

Key words: greenhouse; substrate; electrical conductivity; four-electrode method;

digital agriculture

\section{INTRODUCTION}

Substrate cultivation is a common type in greenhouse. Greenhouse substrate has several good properties for crop growth. It not only can well hold water and nutrition to provide nice conditions for growth of plant's root, but also posses next advantages such as cheaper, rich resource, ventilating, 
easy management, and anti-disease. In recent years, many studies have indicated that substrate electrical conductivity (EC) included plenty of information reflecting substrate properties ${ }^{[1,2]}$. As the introduction of precision farming, it has become more important to understand the condition of substrate EC in a greenhouse. It would help us to know the status of plant growth, and then to perform precision management for the crops in greenhouse. Hence, we developed a portable substrate EC detector, and then used it to evaluate substrate properties in greenhouse, and investigated the effect of water content and salinity on substrate EC.

\section{DEVELOPMENT OF A PORTABLE SUBSTRATE EC DETECTOR}

Figure 1 shows the structure of the portable substrate EC detector. It was based on four-electrode method ${ }^{[3,4]}$. When using this detector to measure substrate EC, it was unnecessary to sample and stir substrate, and was able to continuously measure at some site. The detector included four electrodes, two electrodes connecting with a constant electrical current source and other two electrodes connecting with a voltage meter. The current electrodes injected a steady current into substrate, and then the voltage electrodes detected the output voltage drop between two points of the substrate. Finally, the substrate EC could be calculated according to the voltage drop.

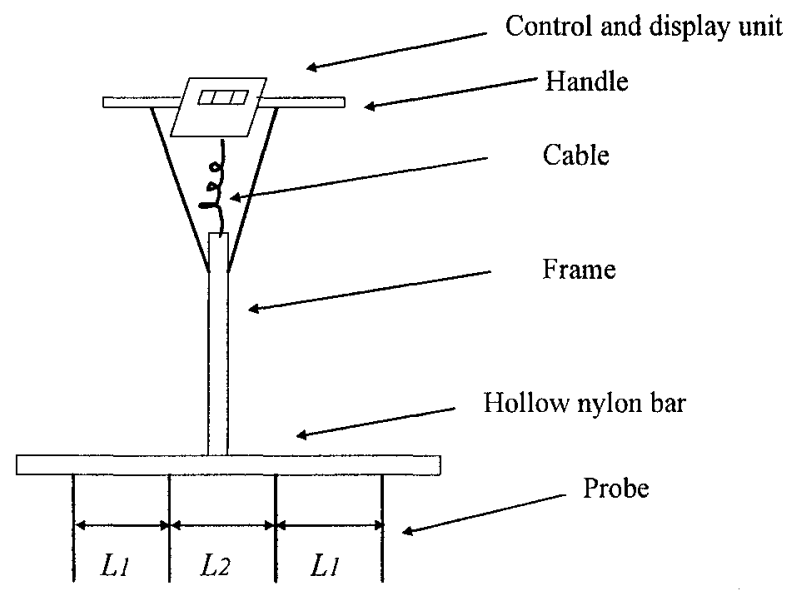

Figure.1 The structure of the portable substrate EC detector

Designed structure of the detector was suitable to expediently using in greenhouse. The probes were made from stainless steel, and were fixed in a 
frame made of a hollow nylon bar with a bundle of wire inside. The probes were connected to the control and display unit by this bundle of wire. Both $L_{1}$ and $L_{2}$ were the optimized structure parameters of the detector, $L_{1}=15 \mathrm{~cm}$ and $L_{2}=20 \mathrm{~cm}$. The weight and height of the EC detector were $4 \mathrm{Kg}$ and $112 \mathrm{~cm}$, respectively. The control and display unit was placed on the top of the detector. It performed next functions: providing a constant electrical current to probes, acquiring substrate $\mathrm{EC}$ data, processing measurement data, recording the data in RAM, and displaying the measured result.

\section{PERFORMANCE TEST OF THE DETECTOR}

\subsection{Materials and methods}

The used substrate was a kind of mixture of vermiculite and straw charcoal. The vermiculite, a kind of inert mineral material, is a compound of $\mathrm{Al}, \mathrm{Mg}, \mathrm{Fe}$ and $\mathrm{Si}$. It is light and can form many big holes when used in greenhouse cultivation so that it has good quality of water-sopping, waterholding, and ventilating. The straw charcoal is composed of semidecomposed vegetables. The texture of the vegetable charcoal is delicacy. It also has rich organic material and nutritional which is necessary for plant growth. The substrate used in this greenhouse test was mixture of vermiculite and straw charcoal with the proportion of 1:1.

As the contrast, the EC of the substrate's solution was also measured with a portable EC tester (DDB303A, Shanghai Precision Scientific Instrument Co.LTD) since there was a linear relation between substrate bulk EC and substrate solution EC. In order to obtain substrate samples with different $\mathrm{EC}$ level, $\mathrm{KCL}$ solution was added into test substrate gradually to change its EC level.

\subsection{Performance test}

In order to establish standard method to real-time measure substrate EC, and to evaluate the performance of the portable detector under the different conditions, three kinds of tests were designed.

(1) According to the reference 4, when the culture medium material was soil, $30 \%$ of water content was a turning point. When the soil water content was below $30 \%$, it had a remarkable effect on soil EC; while when the soil 
water content exceeded $30 \%$, the effect decreased distinctly. Therefore, we could conclude that there might be a similar turning point in substrate. Hence, in the first test we planed to change the water content of the substrate with unchanged salinity to find out the relationship between water content and substrate EC, and then to investigate the measurable range of the portable detector.

(2) When the substrate water content was above the turning point, that is when the water content had little effect on the substrate EC, we would add $\mathrm{KCl}$ solution into the substrate to change its $\mathrm{EC}$ level to establish the estimation model of the substrate EC based on the detector output voltage.

(3) When the substrate water content was below the turning point, that is when the water content had greater effect on the substrate EC, we would add $\mathrm{KCL}$ solution into the substrate to change its $\mathrm{EC}$ value and then to reveal the relationship between substrate $\mathrm{EC}$ and detector output voltage.

\subsection{Result and discussion}

Figure 2 shows the result of the test 1 . The salinity content was $0.326 \%$. Sixteen Experiments in different level of water content were performed. The output voltage of the sensor changed regularly with the change of substrate water content. Due to the output voltage represents the substrate EC value, it was observed that the $50 \%$ of water content was the turning point. It implied that when the substrate water content was below $50 \%$, it would have a remarkable effect on substrate EC. However, when water content was above $50 \%$, the effect of substrate moisture on substrate EC would become very weak. When substrate water content was below $50 \%$, it was necessary to consider the effect of both salinity and water content on substrate EC when using $\mathrm{EC}$ to evaluate the physical and nutritional properties of substrate. When substrate water content was above $50 \%$, the substrate EC value measured by developed portable detector was affected only by ion amount in substrate, and the contribution of water content could be ignored.

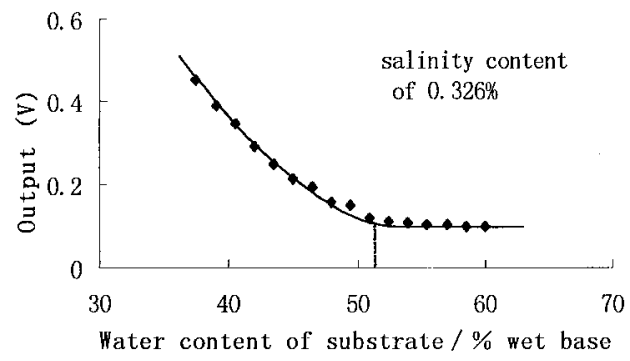

Figure.2 Relationship between substrate moisture and detector output voltage 
In test 2, keeping substrate water content unchanged and in higher level (about $65 \%$ ), and adding $\mathrm{KCl}$ solution to the substrate to change substrate EC level. Twenty-five Experiments in different EC levels were executed. Figure 3 shows the test result. The $\mathrm{X}$ axis is the output voltage measured by the detector. The $\mathrm{Y}$ axis is the substrate solution EC. The voltage output increased regularly as the substrate solution EC decreased. In order to further analyze the correlation between the output voltage and $\mathrm{EC}$ of soil solution, a power function model was attempted. It was observed that almost all the data points distributed closely around the curve. The coefficient of determination $\left(R^{2}\right)$ reached 0.970 . It indicated that the developed detector could estimate substrate electrical conductivity with high accuracy.

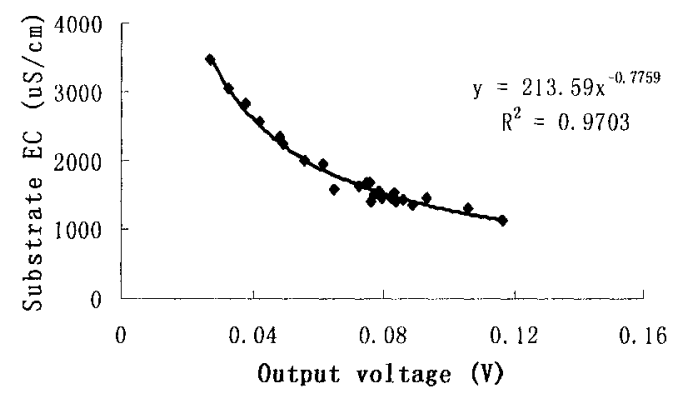

Figure. 3 The relationship between voltage drop and substrate EC in test 2

The objective of the test 3 was to further investigate the performance and precision of the developed EC detector under the lower water content condition. Keeping substrate water content unchanged and in the level of about $45 \%$, and adding $\mathrm{KCl}$ solution to the substrate to change substrate EC. Thirty-two experiments in different EC levels were conducted. Figure 4 shows the result of the test. A power function model was introduced in Figure 4. It was observed that the distribution of the data points in this test was similar to test 2 , and all the data points distributed closely around the curve. The coefficient of determination $\left(\mathrm{R}^{2}\right)$ reached 0.875 . It indicated that the developed detector was also practical to measure substrate EC under the lower water content condition.

Comparing Figure 3 with Figure 4, it could be find out that when the substrate water content was below $50 \%$ although the correlation between substrate EC and output voltage still conformed to the power function relation, the curvature was much less than the situation when substrate moisture content was above $50 \%$. It seems better to use linear model to describe the points shown in Figure 4. The coefficient of determination still 
reached 0.83 when using linear model, and the precision met the requirement of the greenhouse planting. The difference between Figure 3 and Figure 4 could be attributed to different water content. When the water content was below $50 \%$, the substrate EC would be influenced by both water content and total ion amount. It was necessary to design much more performance experiments to find out the variation law of the substrate EC, and further to expand the measurable range of the developed detector.

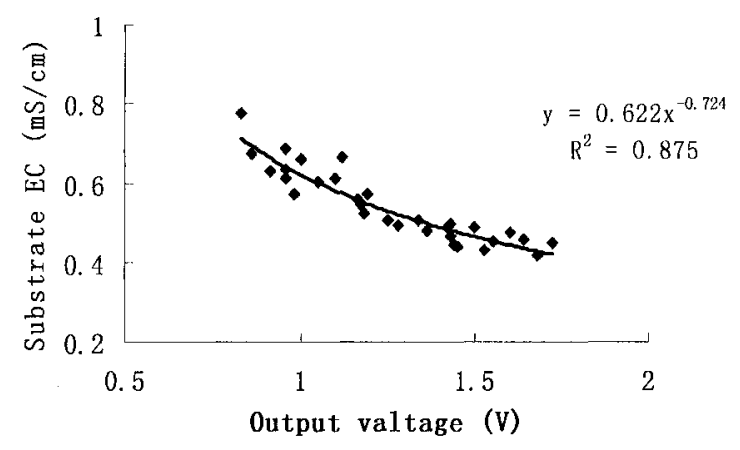

Figure. 4 Relationship between voltage drop and substrate EC in test 3

In a word, all results showed that the developed real-time detector was satisfactory in both accuracy and practicality, and it was able to provide a base for modeling the substrate EC.

\section{CONCLUSIONS}

A portable EC detector was developed. And then it was used to investigate the correlations between substrate EC and two substrate parameters, water content and salinity in greenhouse. The obtained conclusions are as follows:

(1) Developed detector was reliable and easy to operate, and had high precision. It was valuable in precision management of greenhouse cultivation.

(2) Keeping the salinity (ion amount) unchanged and changing the substrate water content, the correlation between substrate water content and substrate EC was analyzed. The result shows that, when the substrate water content was below $50 \%$ the substrate EC was affected by both ion amount and water content. Thus it was necessary to consider the influence of the 
water content when using substrate $\mathrm{EC}$ to evaluate the physical and nutrition properties of the substrate. While the influence could be ignored and the EC was mainly affected by the total ion amount when the water content was above $50 \%$.

(3) Keeping the substrate water content unchanged at about $65 \%$ and changing substrate EC, the output of the developed detector was observed. The result shows that, there was a power function relationship between the output and the substrate $\mathrm{EC}$, and the coefficient of determination reached to 0.970 . When substrate water content was above $50 \%$, the developed model could be used in practice directly.

(4) Keeping the substrate water content unchanged at about $45 \%$ and changing the substrate EC, the output of the detector was also observed. A power function model and a linear function model were used to describe the relationship between the output and the substrate EC, respectively. All models showed significant.

\section{ACKNOWLEDGEMENT}

This study was supported by National High Technology Research and Development Program of China (863 Program): Research and Demonstration for Digital Agriculture (2003AA209040).

\section{REFERENCES}

[1] Naiqian Zhang, Kyeong-Hwan Lee, et al. Simultaneous Measurement of Soil Water content and Method Salinity Using a Frequency-Response, 2004 CIGR International Conference.11-14 October 2004, Beijing, China.

[2] Kitchen N R,Sudduth K A,Drummond S T. Soil electrical conductivity as a crop productivity measure for clay pan soils[J]. J Prod Agric, 1999, 12: 607-617.

[3] Li Minzan, Wang Qi, Wang Maohua. Testing and modeling of soil EC sensor[J]. Transactions of the CSAE, 2004, 20(1): 51-55.

[4] Sun Yurui, Wang Maohua. A Mathematical Model and Its Experimental Study for a Kind of Measurement Method of Soil Electric Conductivity.

Transactions of the CSAE (in Chinese), 2001, 17(2): 20-2.

[5] Corwin D. L. and S. M. Lesch. 2003. Application of Soil Electrical Conductivity to Precision Agriculture --Theory, Principles, and Guidelines. Agronomy Journal 95(3): 455 471. 